\title{
The Stem Cell Niche as the Key to Early Cancer Development
}

\section{Julio Roberto Cáceres Cortés ${ }^{1 *}$}

${ }^{1}$ Laboratory of Cancer and Hematopoiesis, Graduate School of Medicine, National Polytechnic Institute, Mexico

\begin{abstract}
Cancer cells are remarkable for their multiform character. It is now apparent that for normal cells to become cancerous, they shall not have reached a high degree of differentiation and must be properly nourished. Another important factor in the transformation of a normal cell is probably the alteration of the microenvironment that otherwise would hold the cell in check. Loss of niche control over normal stem cells could allow for the reversion of differentiation to a less specialized form, leading to unrestrained growth. The tumor microenvironment consists of the properties conferred by abnormal interactions between tumor and host cells. The aim of the current review is to map out the steps leading to this abnormal interaction process. If indeed the cancer stem cell niche is key to unrestrained proliferation, insights into the alteration in the microenvironment should certainly provide clues as to more effective cancer therapy.
\end{abstract}

Keywords: Stem cell niche; Tumor microenvironment; Stem cells

\section{Introduction}

Cancer is a complex group of diseases with many possible causes, including genetic factors, lifestyle factors, certain types of infections, and environmental influences. The International Agency for Research on Cancer (IARC), a part of the World Health Organization (WHO) http://www.who.int/en/, has now classed air pollution in the same category as tobacco smoke, UV radiation and plutonium. The actual number of people who are diagnosed and who die of cancer each year has indeed grown - because the population is growing larger, and is aging. Cancer is more common among the elderly, so more cases are to be expected as the average age increases. While the data are familiar to most, it may be helpful to review them again. According to the WHO, worldwide cancer-related deaths will likely increase from the 7.8 million in 2008 to over 13 million in 2030. Nearly $30 \%$ of all cancer deaths are directly related to risk factors related to life style and diet, such as overweight and obesity, low consumption of fruits and vegetables, lack of physical exercise, high alcohol consumption and/or smoking. The latter risk factor is the most important, leading to $22 \%$ of cancer mortality worldwide. The "behavioral risk factors" responsible for these deaths may induce niche-deterioration or alteration of the place of residence of the stem cells, which in turn would modify the stem cell response to the microenvironment.

Stem cells change according to the environment in which they are located

Stem cells are characterized by their capacity to perpetuate themselves through self-renewal, differentiate and generate mature cells of a particular tissue [1]. Stem cells reside within most tissues throughout the lifetime of a mammalian organism [2]. To maintain their capacity for division and differentiation, these cells require extensive and precise regulation provided by the local environment or niche surrounding the cell [3]. The influence of the niche on the growth, differentiation and proliferation of stem cells could have important implications for research to combat cancer.

It has been demonstrated that embryonic cells, when proliferating in tissue culture, show no tendency to arrange themselves in a glandular structure as tubes and columns of cells. The result is merely a mass of proliferating elements. As expected, there seems to be no limit to the potential for cell growth [4]. Years later multiplication is as rapid as it was at first, and the general character of the culture and the cells themselves is not altered during this period.

However, if an extracellular matrix is added, the picture changes entirely and the cells take on a distinct organoid arrangement. Growth of embryonic cells is apparently unlimited, as in tissue culture, but alteration and differentiation are dependent upon the influences of the surrounding medium [5].

These distinct results, depending on the microenvironment of embryonic cells, is evidence of the now recognized versatility of stem cells and their ability to change according to the niche in which they are located [6]. The influence of the niche environment on stem cell growth and proliferation could be of interest in various areas of research, including leukaemia and other so-called blood diseases, as well as many types of cancer. Leukemic hematopoietic stem cells reside in systems of production ("niches") that provide the structural and physiological conditions for their growth and survival (Figure 1 and 2).

Leukaemia, part of a broad group of diseases known as hematological neoplasms, is characterized by an abnormal increase in the number of blast cells. Upregulation of survival genes and aberrations in genes regulating cell growth are the common initiating events. Genes commonly mutated during these events include the receptor tyrosine kinases FLT3 or KIT in acute myeloid leukemia (AML) [7], TP53 or RB1 in chronic myelogenous leukemia (CML) [8], and NOTCH1 in acute lymphoblastic leukemia (ALL) [9]. Each of these genes can acquire a variety of different mutations and each hematological neoplasm itself can acquire mutations in multiple genes.

Accumulating evidence suggests that stem cell behavior is regulated

*Corresponding author: Julio Roberto Caceres-Cortes, Laboratory of Cancer and Hematopoiesis, Graduate School of Medicine, National Polytechnic Institute, Plan de San Luis and Diaz Miron s/n, Col. Casco de Santo Tomas, Miguel Hidalgo, CP 11340, Mexico, DF Tel: +5255 5729-6000; Fax: +5255 53411600; E-mail: cortesj@unam.mx

Received July 19, 2013; Accepted October 27, 2013; Published October 29 2013

Citation: Cáceres Cortés JR (2013) The Stem Cell Niche as the Key to Early Cancer Development. J Cytol Histol 4: 188. doi: 10.4172/2157-7099.1000188

Copyright: ( 2013 Cáceres Cortés JR. This is an open-access article distributed under the terms of the Creative Commons Attribution License, which permits unrestricted use, distribution, and reproduction in any medium, provided the original author and source are credited. 
by both extracellular signals from the cell niche and intrinsic signals within stem cells [10]. Recent observations indicate that mesenchymal stem cells can create a cancer stem cell niche to elicit a stem celllike phenotype in carcinoma cells, which in turn allows for tumor progression [11]. Among the repertoire of stimuli in such a niche are soluble chemical signals and a number of physical components of the microenvironment. The bottom line is that the stem cell microenvironment is involved in regulating the normal or abnormal fate of the stem cell with respect to self-renewal, quiescence, and differentiation [12].

Numerous examples of this mode of regulation indicate that biological aspects of the niche must be studied. For example, a variety of interactive signaling networks become altered in organ stem cells and in their differentiated niches during the aging process. Remarkably, the behavior of old stem cells can be rejuvenated in "young" environments [13].

Moreover, in response to signals from the microenvironment, in vivo bone marrow stem cells (as well as those from other sources) can become differentiated cells when implanted into diverse organs. Optimization of cell implants should enhance the clinical significance of adult stem cell-based regenerative therapy [14]. One of the first clinical applications of stem cells was the autologous transplantation of a dental follicle performed by Dr. L. Garcia, who observed the generation of a new first molar tooth from the third molar follicle in a boy (Vision Cuba,August 31, 1956). This seems to have occurred due to influences set up by environmental conditions of the new tooth socket rather than because of an inherited control in the cells themselves. Indeed, the ability of stem cells for a site directed differentiation and functional integration is known to depend on extrinsic factors [15]. Studies on stem cells show that they continue to self-renew because of a set of transcription factors that prevents their differentiation and promotes their proliferation, and because of epigenetic processes that might be under the control of the stroma [16]. Surprisingly, the processes of selfrenewal and differentiation of stem cells can be reversed by inducing pluripotency through the introduction of proteins, miRNAs or other factors [17]. In experimental studies, classic fusion between somatic cells and embryonic carcinoma, embryonic stem, or embryonic germ cells resulted in reprogramming. This demonstrates that pluripotency is dominant over differentiation $[18,19]$.

Currently several components required for stem cells growth, for example, have been identified (Figure 2). Among them basic fibroblast growth factor (bFGF) has been shown to be essential for self-renewal in embryonic stem cells $[20,21]$. Three other requirements are (i) feeder cells, conditioned medium, or cytokines, such as TGF $[22,23]$ or Wnt3a [24]; (ii) matrix; and (iii) FBS or serum replacement [25,26].

The in vitro proliferation of cells from mammalian tissues is stimulated by specific growth factors and is assumed that these growth factors act in vivo as paracrine or autocrine regulators of normal cell production. In several pathological conditions such as cancer, it has been proposed that the autocrine production of growth factors is an important element of the tumorigenic process [27,28]. Although autocrine growth factor production may cause hiperplasia, malignancy almost certainly involves several independent molecular perturbations, which combine to cause the uncontrolled proliferation of a small number of mutant stem cells [29]. Whilst in some experimental systems, single gene products such as E1a, ras and myc can convert immortalized cells to malignant cells, in most spontaneous tumors, it is not possible to identified all of the molecular lesions relevant to the malignant phenotype. For particular tumors, such as chronic myeloid

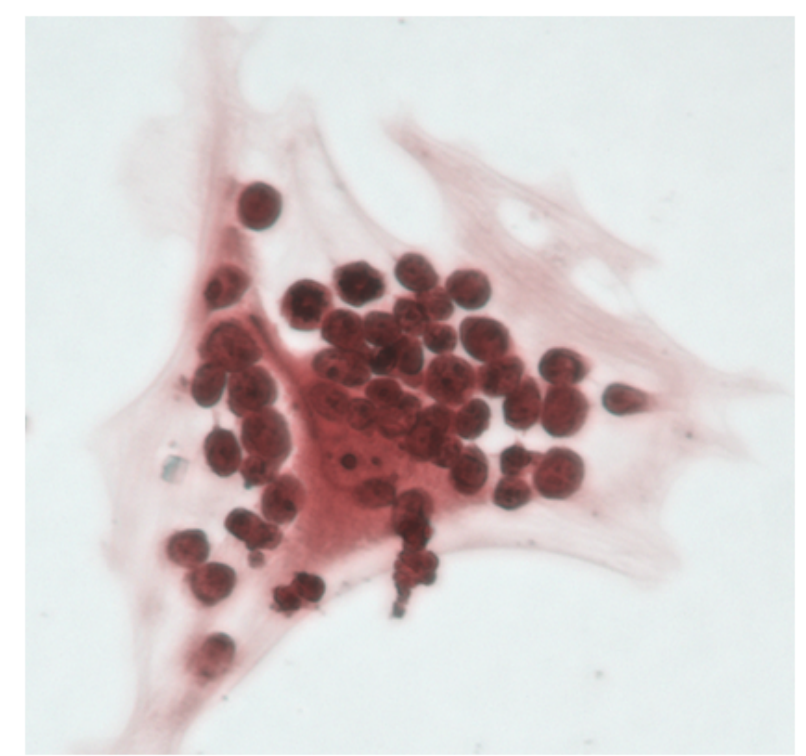

Figure 1: A group of mouse leukemic T cells NOTCH1+ in a culture medium that provides a niche mimicking in vivo conditions. Leukemic cells are in the presence of IL-7 and FLT3, and have an intimate relationship with thymus stromal cells. Magnification: 400X. (Caceres-Cortes JR and Hoang T; IRICMontreal, Canada).

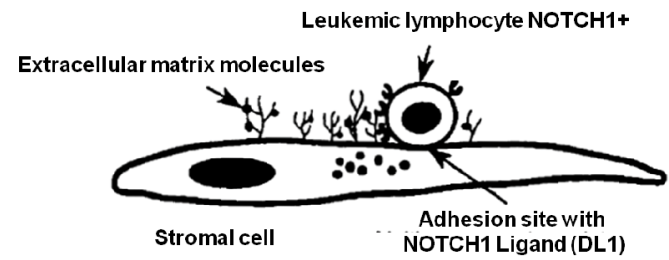

Multi-component regulatory niche system

1. Heterogeneity of stromal cells

2. Types of growth factors produced

2. Types of growth factors

4. Matrix molecules

Figure 2: Schematic diagram of picture in Figure 1 showing the interaction between stromal cell, target cell and growth factor.

leukemia (CML) or pancreatic carcinoma, the frequency of specific lesions (the Philadephia chromosome or activation of the ras gene, respectively) approaches $100 \%$. In lymphocytic leukemia NOTCH1, $S C L$ and $L M O$ genes are considered to be capital for cell expansion in transgenic mouse [30]. Thus, it is reasonable to assume that these lesions are involved in either the initiation or maintenance of the malignant phenotype. On the other hand the role of the microenvironment in the pathophysiology and progression of preleukemia or latency period has remained controversial, although there is evidence that stroma and matrix components, and their interactions with clonal cells, play an important role through inhibition of programmed cell death (apoptosis) but allowing autophagy [31].

\section{Cancer is an abnormality of growth}

Cancer is regarded as a specific malady that can be found in almost every tissue of the human frame, including skin, mucous membranes, muscles, fibrous tissues, nerves, and medullary cavities of the bones. It 
has a marked tendency to spread in all directions, and to return after being removed, either in the place it originally occupied or in some other part of the body. It eventually induces decay and dissolution in the whole animal economy. Cancer can be caused by any factor that tends to increase the proliferation and motility of cells particularly sensitive but not yet cancerous. Such a factor, which can act either by chemical or physical means, is not essentially different from the growth stimuli that lead to the regeneration of tissue. In some cases there appear to be additional factors present in certain cells that make them particularly responsive to sensitizing substances coming from other organs.

The elements of every cancerous growth are cancer cells intermixed with the structure of various types of tissue (extracellular matrix and various cell types, including fibroblasts, endothelial cells and infiltrating leukocytes). Morra et al. describes the terminology used to describe the tumor microenvironment. For instance, tumor stromal cells consist of "tumor-associated endothelial cells" (TECs), "cancer-associated fibroblasts" (CAFs), "tumor-associated macrophages", and distinct sets of "tumor-directed lymphocytes" [32].

The tumor niche has now emerged from the reductionist point of view of being a collection of a few cells to becoming the source of prolific production of cancer stem cells [33]. Over the past few years, improved understanding of the molecular signalling pathways required to sustain tumor growth is beginning to unravel the complexity of the tumor microenvironment. Immune cells may account for as much as $50 \%$ of tumor mass. Tumor-secreted MCP1 attracts bloodcirculating monocytes, which then differentiate into tumor-associated macrophages (TAMs) following exposure to the cytokine MCSF in the tumor microenvironment. TAMs are generally thought to exert protumorigenic functions by having poor antigen-presenting abilities, suppressing Th1 adaptive immunity and promoting wound healing. This results in the substitution of natural tissue by new cancerous tissue. It is considered that TAMs exert their pro-tumorigenic effects mainly through signaling mechanisms that: (a) promote angiogenesis through the secretion of VEGF and PDGF, (b) induce tumor growth through the production of TGF $\beta$, and (c) enhance cancer cell migration through the production of matrix metalloproteases, particularly MMP9 $[34,35]$.

An increasing list of signalling molecules produced by immune cells that contribute to the promotion of tumor growth has been discovered such as EGF, VEGF, FGF2, chemokines and cytokines, cysteine cathepsins, heparanase and matrix metalloproteinases. Production of these signalling molecules is found to direct angiogenesis, tumor invasion and promote cellular proliferation. In pancreatic ductal adenocarcinoma, tumor derived GM-CSF is necessary to drive the recruitment of Gr-1+CD11b+ cells, which in turn inhibit antigenspecific T cells. Furthermore, VEGF is known to sustain tumor growth through the inhibition of dendritic cell maturation, thus enhancing tumor survival and growth [36].

Solid tumors in both humans and animals are hypoxic environments which affect tumor cell metabolic pathways and surrounding tissue environments. Hypoxic tumor microenvironments are also found to increase resistance to cytotoxic anti-cancer treatment and radiation therapy, thus resulting in poor clinical prognosis. Mutations in key oncogenes such as MYC, p53 and AKT are found to affect cellular metabolism [37]. In addition, HIF1 regulates the hypoxic tumor environment through the upregulation of pyruvate dehydrogenase 1 , which leads to a decreased amount of pyruvate entering the citric acid cycle and decreased mitochondrial oxygen consumption [38,39].

To become cancerous, a cell shall not have reached a high degree of differentiation and must be properly nourished. Moreover, its niche must be altered. Insights into the influence of the microenvironment can be obtained only through observations of its effects upon cells, such as when the niches are disturbed. Observations of this kind have revealed that the first alteration in the environment that normally holds cell proliferation in check is of a chemical nature (chemo-differentiation), followed by physical and/or other chemical stimuli. Chemical changes can be brought about in several different ways, including by hormones, trophic and neurovascular factors, and by disturbance of normal tissue continuity. However, exactly how this influence is exerted remains a mystery [40].

Since we can discard all ideas of specificity and autonomy, the question is not why certain cells of the body take on a peculiar and particular neoplastic tendency. Rather, it is how a loss of the normal environmental control leads certain cells to differentiate and grow in an unrestrained manner. It is known that cancer cells reprogram adjacent stromal cells so that the latter will optimize the environment by regulating energy metabolism to fuel cell growth and division $[41,42]$. In an invaluable contribution, Wallace DC [43] drew attention to certain mutations in mitochondrial genes that alter rather than inactivate mitochondrial energy metabolism by modulating signal transduction pathways, transcriptional circuits and chromatin structure for cell survival. At the molecular level, first-hit genetic (or epigenetic) alterations are thought to initiate a pre-malignant process by interacting with the existing machinery of the physiological cell state [44]. Additional second-hit mutations are necessary to drive the transformation [45].

\section{Cancer as a universal cell property}

The concept of cancer stem cells has rapidly gained credibility over the past 10 years. These cells are considered to possess properties similar to those described for tissue stem cells: self-renewal and asymmetric division resulting in the generation of daughter cells destined to differentiate, enabling the regeneration of a tissue [46,47] The hallmark of a cancer cell is its multiform character, being able to: a) sustain proliferative signaling, b) evade growth suppressors, c) resist cell death, d) enable replicative immortality, e) induce angiogenesis, f) activate invasion and metastasis, $g$ ) reprogram energy metabolism, and h) escape immune destruction.

The view that unrestrained and malignant proliferation is a universal cell property is supported by a vast body of knowledge accumulated through many years. Each tumor is originally derived from a transformed cell. Thus, most tumors (but perhaps not all) are believed to have a clonal origin. Later, subclones often develop as the tumor grows. Evaluation of this process is of relevance in carcinogenesis. Five lines of evidence are herein provided in support the idea of clonality in human tumors:

1. The presence of the same chromosome marker in all cells of a single tumor. The identification of the Philadelphia chromosome in erythropoietic and granulopoietic cells provides clear evidence that human chronic granulocytic leukaemia originates from pluripotent stem cells [48].

2. The cells of a tumor of plasma cells produce the same type of immunoglobulin. Plasmacytomas express the same idiotype and rearrange the same light $(\mathrm{V}(\mathrm{L}))$ and heavy $(\mathrm{V}(\mathrm{H}))$ variable region genes to express a characteristic monoclonal antibody [49].

3. Tumor cells have common antigens [50]. 
4. Although normal tissues of a woman are a mosaic of cells in which one $\mathrm{X}$ chromosome or the other has been inactivated, all her tumor cells - even if from multiple sites- have the same X chromosome inactivated. In each female somatic cell, either the paternal or maternal X-chromosome is randomly inactivated in early embryogenesis (the Lyon hypothesis). Several loci have been used to assess skewed X-chromosome inactivation, including the phosphoglycerate kinase $(P G K)$ gene, the hypoxanthine phosphoribosyltransferase (HPRT) gene and the DXS255 locus. Today, most published X-chromosome inactivation studies make use of the human androgen receptor (HUMARA) gene. Tumors with identical allelic inactivation patterns are of monoclonal origin [51,52].

5. Most solid tumors, to a greater or lesser degree, resemble the tissues from which they arose, and not a tissue from a disparate cell lineage [53].

Once we accept the natural tendency to growth and its restraint by environmental influences, we get quite a different perspective on many biological problems. That is, the burden of explanation is placed on a different phase of the problem. The important question is no longer why organisms grow, but instead why they stop growing. Thus, rather than ask why a wound heals, we should explore why the healing growth ceases once the defect has been repaired. Likewise, we need not wonder why we get tumors. The more logical question is why they are not much more common than experience proves to be.

\section{Self-eating and self-killing: Simultaneous induction}

Autophagy is an evolutionarily conserved process involved in microenvironmental adaptation and tumor development [54]. Recent evidence in mice suggests that autophagic defects in haematopoietic stem cells are implicated in leukaemia. Indeed, mice lacking Atg7 (Autophagy-related protein 7) in haematopoietic stem cells develop an atypical myeloproliferation resembling human myelodysplastic syndrome, which progresses to AML. Studies suggest that the accumulation of damaged mitochondria results in cell transformation. Interestingly, bone marrow cells from preleukaemic patients are characterized by mitochondrial abnormalities and increased rates of cell death. A role of autophagy in the transformation of normal cells into cancerous cells has been proposed in other types of cancer [55].

Bialik and Kimchi reported that autophagy has been harnessed by some tumor cells as a survival mechanism to protect them against apoptosis-inducing signals [56]. Chiacchiera and Simone reported that autophagy, or self-cannibalization, is a pro-survival mechanism for cancer cells that emerge under conditions imposed by nutrient or growth factor deprivation [57]. The cytoprotective role of autophagy following chemotherapy has been confirmed by other investigators $[58,59]$.

Although apoptosis and autophagy are separate processes, they both are controlled by the Bcl-2 protein [60]. Apaf- 1 (apoptotic protease activating factor 1) and Beclin-1 (mammalian orthologue of yeast Atg6) have been identified as Bcl-2-connected proteins, suggesting that cell death is operated by a mechanical system. This system includes components that contain mechanical actuators: [Bcl-2]-[Apaf1] in apoptosis and [Bcl-2]-[Beclin1] in autophagy. Each duo programs the characteristics of operation of the molecules that assemble in living cells and disassemble in dying cells [61]. Stem cells enter in autophagy more efficiently than precursors or differentiated cells do probably because [Bcl-2]-[Beclin1] dissociation is preferably controlled [62]. Autophagy and apoptosis can be triggered by common upstream signals, and sometimes they occur at the same time in separate cell populations. However, this could also be explained by how cells divide. Symmetric cell division is defined as the generation of daughter cells destined to acquire the same cell characteristics. On the other hand, stem cells divide asymmetrically to generate one daughter cell with stem-cell and one with differentiated-cell characteristics. The normal differentiation of progenitor cells into lineage-specific cells results in a loss of multilineage potential and follows a hierarchical pattern $[63,64]$.

As in many tissues, rare populations of cancer stem cells have been characterized in AML. The ability of stem cells to undergo both asymmetric and symmetric cell division is what defines them as stem cells. Thus a key question is how stem cells regulate their self-renewal and multipotency properties. It is proposed that stem cells lose the potential for continued self-renewal when removed from their normal cellular environment, the so-called stem cell niche, suggesting an essential role for the microenvironment in controlling stem cell behavior [65].

It has been demonstrated that stem cell factor (SCF) is a growth factor situated in the stem cell niche that sustains cell survival and selfrenewal, and therefore asymmetric division [66]. The presence of SCF in the haematopoietic microenvironment is particularly important. Exposure of haematopoietic stem cells (HSC) to different concentrations of soluble stem cell factor is one mechanism that can regulate HSC selfrenewal divisions in vitro $[67,68]$. SCF is also an important, but not selective, physiological regulator of HSC activity in vivo $[69,70]$.

Interestingly, many properties of HSCs change during development, such as a marked decrease in SCF sensitivity [71]. We have tested the inhibition of c-Kit signaling to induce programmed cell death, finding the result to be simultaneous combinations of apoptosis and autophagy. Further, quantification of cell populations revealed an additive phenomenon, consisting of a small number of cells in autophagy and a greater number in apoptosis [72]. Hence, specific inhibition of c-Kit signaling reveals the importance of this protein for cell survival.

Knoblich (2008) provides detailed experimental results for stem cells undergoing asymmetric and symmetric cell division to generate progeny, finding an asymmetrical distribution of many proteins, membrane compartments, organelles and transcription factors, as well as some DNA between the two daughter cells [73]. Moreover, differentiation requires up-regulation of the genes associated with the adopted pathway and silencing of genes that specify the alternate pathway. This unique gene expression profile in the "mature cells" is tightly regulated by the action of several different transcription factors and growth factors.

\section{A survival strategy in stem cells: Autophagy triggered by DNA damage}

Although cell responses aimed at repairing DNA damage are crucial for maintaining the integrity of the genome, they must also be held in check to prevent erroneous activity like "repair" of replicationassociated breaks. Recent studies suggest a role of autophagy together with the post-translational modification of a key DNA repair protein Sae2 (human CtIP) in keeping this process under control.

Our body cells are constantly being aggravated by potential mutagens, including UV radiation, genotoxic chemicals, ionizing radiation, and byproducts of cell metabolism (e.g., reactive oxygen species and lipid peroxidation). In order to maintain the genomic integrity essential for survival, cells propagate signals in response to the detection of DNA damage and its potential for repair. 
Autophagy, a selective degradation of cellular components, one of these signals has been shown to play a role in a wide variety of normal physiological processes, including energy metabolism, organelle turnover, growth regulation and aging. Indeed, the in vivo effects of Beclin-1 haploinsufficiency on autophagy have proven to be determinant in the onset of cancer [74]. Many of the proteins involved in the DNA damage response are acetylated, and the purpose of this modification was recently clarified [75]. It is now understood that acetylation is involved in regulating the DNA damage response via an autophagic process [76,77].

The acetylated double strand break repair protein, Sae2 (human CtIP), is removed from the nucleus and degraded via autophagy. Rapamycin, which stimulates autophagy by inhibiting Tor, also causes Sae 2 degradation. This presumably allows the DNA damage response machinery to be sidetracked from actively replicating DNA, a process that normally contains naturally occurring breaks. Mistakenly "repairing" replication associated breaks could result in potentially detrimental DNA damage.

It is possible that repair activity through an acetylation dependent autophagic process, would be also present in cancer cells. The usual process of autophagy, an action plan to prevent further damage to DNA after an initial insult, may be used by cancer stem cells for their own benefit.

\section{Conclusion}

It seems likely that the stem cell niche is key to early cancer development. Here I have given instances of two murine costimulatory receptor-ligand pairs: NOTCH1/DL1 and c-Kit/SCF. Over the past few years, improved understanding of the molecular signaling pathways required to sustain tumor growth has begun to unravel the complexity of the tumor microenvironment. Hematopoietic stem cells are controlled by both intrinsic (transcription factors, signal transduction pathways) and extrinsic regulators (niche factors). The tumor microenvironment is defined by the abnormal interactions between tumor and host cells, often characterized by hypoxia, nutrient deprivation, acidosis and aberrant stroma. At the level of survival, cancer cells can mobilize autophagy in response to various stimuli, suggesting that they can also exploit autophagy for their own benefit. Whereas normal cells have evolved a number of mechanisms for protecting DNA from the constant onslaught by potential mutagens, cancerous cells appear to have sequestered autophagy machinery to protect mutations by regulating the activity of DNA repair enzymes.

\section{Conflict of Interest Statement}

This work was conducted in the absence of any commercial or financial relationships that could be construed as a potential conflict of interest.

\section{Acknowledgments}

The author's own work was supported by a grant from the National Science and Technology Council of Mexico (CONACYT) 202282 and The National Polytechnic Institute (IPN)-SIP 20130984.

\section{References}

1. Reya T, Morrison SJ, Clarke MF, Weissman IL (2001) Stem cells, cancer, and cancer stem cells. Nature 414: 105-111.

2. Vestentoft PS (2013) Development and molecular composition of the hepatic progenitor cell niche. Dan Med J 60: B4640.

3. Conway A, Schaffer DV (2012) Biophysical regulation of stem cell behavior within the niche. Stem Cell Res Ther 3: 50 .
4. Darbinyan A, Kaminski R, White MK, Darbinian N, Khalili K (2013) Isolation and propagation of primary human and rodent embryonic neural progenitor cells and cortical neurons. Methods Mol Biol 1078: 45-54.

5. Narayanan K, Lim VY, Shen J, Tan ZW, Rajendran D, et al. (2013) Extracellula Matrix-Mediated Differentiation of Human Embryonic Stem Cells: Differentiation to Insulin-Secreting Beta Cells. Tissue Eng Part A

6. Morsczeck C, Götz W, Schierholz J, Zeilhofer F, Kühn U, et al. (2005) Isolation of precursor cells (PCs) from human dental follicle of wisdom teeth. Matrix Biol 24: 155-165.

7. Luthra R, Patel KP, Reddy NG, Haghshenas V, Routbort MJ, et al. (2013) Next generation sequencing based multi-gene mutational screen for acute myeloid leukemia using miseq: applicability for diagnostics and disease monitoring. Haematologica.

8. Johansson B, Fioretos T, Mitelman F (2002) Cytogenetic and molecular genetic evolution of chronic myeloid leukemia. Acta Haematol 107: 76-94.

9. Lobry C, Oh P, Aifantis I (2011) Oncogenic and tumor suppressor functions of Notch in cancer: it's NOTCH what you think. J Exp Med 208: 1931-1935.

10. Singh SR (2012) Stem cell niche in tissue homeostasis, aging and cancer. Curr Med Chem 19: 5965-5974.

11. Li HJ, Reinhardt F, Herschman HR, Weinberg RA (2012) Cancer-stimulated mesenchymal stem cells create a carcinoma stem cell niche via prostaglandin E2 signaling. Cancer Discov 2: 840-855.

12. Tieu KS, Tieu RS, Martinez-Agosto JA, Sehl ME (2012) Stem cell niche dynamics: from homeostasis to carcinogenesis. Stem Cells Int 2012: 367567.

13. Silva H, Conboy IM (2008) Aging and stem cell renewal. StemBook [Internet] Cambridge (MA): Harvard Stem Cell Institute.

14. Chiu RC (2003) Bone-marrow stem cells as a source for cell therapy. Heart Fail Rev 8: 247-251.

15. Andressen C (2013) Neural stem cells: from neurobiology to clinical applications. Curr Pharm Biotechnol 14: 20-28.

16. Niwa $\mathrm{H}$ (2007) How is pluripotency determined and maintained? Development 134: $635-646$

17. Takahashi K, Tanabe K, Ohnuki M, Narita M, Ichisaka T, et al. (2007) Induction of pluripotent stem cells from adult human fibroblasts by defined factors. Cell 131: $861-872$

18. De Los Angeles A, Loh YH, Tesar PJ, Daley GQ (2012) Accessing naïve human pluripotency. Curr Opin Genet Dev 22: 272-282.

19. van Oosten AL, Costa $Y$, Smith A, Silva JC (2012) JAK/STAT3 signalling is sufficient and dominant over antagonistic cues for the establishment of naive pluripotency. Nat Commun 3: 817.

20. Cao S, Wang F, Liu L (2013) Isolation and culture of bovine embryonic stem cells. Methods Mol Biol 1074: 111-123.

21. Xu C, Rosler E, Jiang J, Lebkowski JS, Gold JD, et al. (2005) Basic fibroblast growth factor supports undifferentiated human embryonic stem cell growth without conditioned medium. Stem Cells 23: 315-323.

22. Beattie GM, Lopez AD, Bucay N, Hinton A, Firpo MT, et al. (2005) Activin A maintains pluripotency of human embryonic stem cells in the absence of feeder layers. Stem Cells 23: 489-495.

23. Amit M, Shariki C, Margulets V, Itskovitz-Eldor J (2004) Feeder layer- and serum-free culture of human embryonic stem cells. Biol Reprod 70: 837-845.

24. Sato N, Meijer L, Skaltsounis L, Greengard P, Brivanlou AH (2004) Maintenance of pluripotency in human and mouse embryonic stem cells through activation of Wnt signaling by a pharmacological GSK-3-specific inhibitor. Nat Med 10: $55-63$.

25. Holden C (2005) Human embryonic stem cells. Getting the mice out of ES cell cultures. Science 307: 1393.

26. Xu C, Inokuma MS, Denham J, Golds K, Kundu P, et al. (2001) Feeder-free growth of undifferentiated human embryonic stem cells. Nat Biotechnol 19 971-974.

27. Onnis B, Fer N, Rapisarda A, Perez VS, Melillo G (2013) Autocrine production of IL-11 mediates tumorigenicity in hypoxic cancer cells. J Clin Invest 123 1615-1629. 
28. Nickerson NK, Mill CP, Wu HJ, Riese DJ 2nd, Foley J (2013) Autocrine-derived epidermal growth factor receptor ligands contribute to recruitment of tumorassociated macrophage and growth of basal breast cancer cells in vivo. Oncol Res 20: 303-317.

29. Reddy KL, Feinberg AP (2013) Higher order chromatin organization in cancer Semin Cancer Biol 23: 109-115.

30. Gerby B, Hoang T (2013) Modelling acute leukemias in mice: clonal evolution and the emergence of leukemic stem cells. BMC Proceedings 7: K1.

31. Kerbauy DB, Deeg HJ (2007) Apoptosis and antiapoptotic mechanisms in the progression of myelodysplastic syndrome. Exp Hematol 35: 1739-1746.

32. Morra L, Moch H (2011) Periostin expression and epithelial-mesenchymal transition in cancer: a review and an update. Virchows Arch 459: 465-475.

33. Liu H, Zhang W, Jia Y, Yu Q, Grau GE, et al. (2013) Single-cell clones of liver cancer stem cells have the potential of differentiating into different types of tumor cells. Cell Death Dis 4: e857.

34. Obeid E, Nanda R, Fu YX, Olopade OI (2013) The role of tumor-associated macrophages in breast cancer progression (review). Int J Oncol 43: 5-12.

35. Fukuda K, Kobayashi A, Watabe K (2012) The role of tumor-associated macrophage in tumor progression. Front Biosci (Schol Ed) 4: 787-798.

36. Robert J (2013) [Biology of cancer metastasis]. Bull Cancer 100: 333-342.

37. Sasaki R, Narisawa-Saito M, Yugawa T, Fujita M, Tashiro $H$, et al. (2009) Oncogenic transformation of human ovarian surface epithelial cells with defined cellular oncogenes. Carcinogenesis 30: 423-431.

38. Koukourakis MI, Giatromanolaki A, Sivridis E, Gatter KC, Harris AL (2005) Pyruvate dehydrogenase and pyruvate dehydrogenase kinase expression in non small cell lung cancer and tumor-associated stroma. Neoplasia 7: 1-6.

39. Enns L, Ladiges W (2012) Mitochondrial redox signaling and cancer invasiveness. J Bioenerg Biomembr 44: 635-638.

40. Polyak K, Haviv I, Campbell IG (2009) Co-evolution of tumor cells and their microenvironment. Trends Genet 25: 30-38.

41. Mitra AK, Zillhardt M, Hua Y, Tiwari P, Murmann AE, et al. (2012) MicroRNAs reprogram normal fibroblasts into cancer-associated fibroblasts in ovarian cancer. Cancer Discov 2: 1100-1108.

42. Yang G, Rosen DG, Zhang Z, Bast RC Jr, Mills GB, et al. (2006) The chemokine growth-regulated oncogene 1 (Gro-1) links RAS signaling to the senescence of stromal fibroblasts and ovarian tumorigenesis. Proc Natl Acad Sci U S A 103 16472-16477.

43. Wallace DC (2012) Mitochondria and cancer. Nat Rev Cancer 12: 685-698.

44. Sagar J, Chaib B, Sales K, Winslet M, Seifalian A (2007) Role of stem cells in cancer therapy and cancer stem cells: a review. Cancer Cell Int 7: 9.

45. Vermeulen L, Sprick MR, Kemper K, Stassi G, Medema JP (2008) Cancer stem cells--old concepts, new insights. Cell Death Differ 15: 947-958.

46. Speck NA, Gilliland DG (2002) Core-binding factors in haematopoiesis and leukaemia. Nat Rev Cancer 2: 502-513.

47. Knudson AG (1996) Hereditary cancer: two hits revisited. J Cancer Res Clin Oncol 122: 135-140.

48. Skorski T (2012) Genetic mechanisms of chronic myeloid leukemia blastic transformation. Curr Hematol Malig Rep 7: 87-93.

49. Diaw L, Siwarski D, Coleman A, Kim J, Jones GM, et al. (1999) Restricted immunoglobulin variable region (lg $\mathrm{V}$ ) gene expression accompanies secondary rearrangements of light chain Ig $\mathrm{V}$ genes in mouse plasmacytomas. J Exp Med 190: 1405-1416

50. Zheng HC, Takahashi H, Murai Y, Cui ZG, Nomoto K, et al. (2006) Upregulated EMMPRIN/CD147 might contribute to growth and angiogenesis of gastric carcinoma: a good marker for local invasion and prognosis. $\mathrm{Br} \mathrm{J}$ Cancer 95 : 1371-1378.

51. Balderman S, Lichtman MA (2011) A history of the discovery of random $x$ chromosome inactivation in the human female and its significance. Rambam Maimonides Med J 2: e0058.

52. Manoukian S, Verderio P, Tabano S, Colapietro P, Pizzamiglio S, et al. (2013) $X$ chromosome inactivation pattern in BRCA gene mutation carriers. Eur $J$ Cancer 49: 1136-1141.
53. Egeblad M, Nakasone ES, Werb Z (2010) Tumors as organs: complex tissues that interface with the entire organism. Dev Cell 18: 884-901.

54. Liang C, Feng P, Ku B, Dotan I, Canaani D, et al. (2006) Autophagic and tumour suppressor activity of a novel Beclin1-binding protein UVRAG. Nat Cell Biol 8: 688-699.

55. Watson AS, Mortensen M, Simon AK (2011) Autophagy in the pathogenesis of myelodysplastic syndrome and acute myeloid leukemia. Cell Cycle 10: 17191725.

56. Bialik S, Kimchi A (2008) Autophagy and tumor suppression: recent advances in understanding the link between autophagic cell death pathways and tumor development. Adv Exp Med Biol 615: 177-200.

57. Chiacchiera F, Simone C (2009) Signal-dependent control of autophagyrelated gene expression. Methods Enzymol 453: 305-324.

58. Katayama M, Kawaguchi T, Berger MS, Pieper RO (2007) DNA damaging agent-induced autophagy produces a cytoprotective adenosine triphosphate surge in malignant glioma cells. Cell Death Differ 14: 548-558.

59. Abedin MJ, Wang D, McDonnell MA, Lehmann U, Kelekar A (2007) Autophagy delays apoptotic death in breast cancer cells following DNA damage. Cell Death Differ 14: 500-510.

60. Marquez RT, Xu L (2012) Bcl-2:Beclin 1 complex: multiple, mechanisms regulating autophagy/apoptosis toggle switch. Am J Cancer Res 2: 214-221.

61. Yip KW, Reed JC (2008) Bcl-2 family proteins and cancer. Oncogene 27: 63986406.

62. Cáceres-Cortés JR (2013) Blastic leukaemias (AML): a biologist's view. Cell Biochem Biophys 66: 13-22.

63. Punzel M, Ho AD (2001) Divisional history and pluripotency of human hematopoietic stem cells. Ann N Y Acad Sci 938: 72-81.

64. Yahata T, Muguruma Y, Yumino S, Sheng Y, Uno T, et al. (2008) Quiescent human hematopoietic stem cells in the bone marrow niches organize the hierarchical structure of hematopoiesis. Stem Cells 26: 3228-3236.

65. Voog J, Jones DL (2010) Stem cells and the niche: a dynamic duo. Cell Stem Cell 6: 103-115.

66. Giebel B, Bruns I (2008) Self-renewal versus differentiation in hematopoietic stem and progenitor cells: a focus on asymmetric cell divisions. Curr Stem Cell Res Ther 3: 9-16.

67. Audet J, Miller CL, Eaves CJ, Piret JM (2002) Common and distinct features of cytokine effects on hematopoietic stem and progenitor cells revealed by doseresponse surface analysis. Biotechnol Bioeng 80: 393-404.

68. Zandstra PW, Conneally E, Petzer AL, Piret JM, Eaves CJ (1997) Cytokin manipulation of primitive human hematopoietic cell self-renewal. Proc Nat Acad Sci U S A 94: 4698-4703.

69. Miller CL, Rebel VI, Lemieux ME, Helgason CD, Lansdorp PM, et al. (1996) Studies of W mutant mice provide evidence for alternate mechanisms capable of activating hematopoietic stem cells. Exp Hematol 24: 185-194.

70. Driessen RL, Johnston HM, Nilsson SK (2003) Membrane-bound stem cell factor is a key regulator in the initial lodgment of stem cells within the endosteal marrow region. Exp Hematol 31: 1284-1291.

71. Bowie MB, Kent DG, Copley MR, Eaves CJ (2007) Steel factor responsiveness regulates the high self-renewal phenotype of fetal hematopoietic stem cells. Blood 109: 5043-5048.

72. Lara-Padilla E, Caceres-Cortes JR (2012) On the nature of the tumor-initiating cell. Curr Stem Cell Res Ther 7: 26-35.

73. Knoblich JA (2008) Mechanisms of asymmetric stem cell division. Cell 132 583-597.

74. Galluzzi L, Kroemer G (2013) Common and divergent functions of Beclin 1 and Beclin 2. Cell Res.

75. Choudhary C, Kumar C, Gnad F, Nielsen ML, Rehman M, et al. (2009) Lysine acetylation targets protein complexes and co-regulates major cellular functions. Science 325: 834-840

76. Robert T, Vanoli F, Chiolo I, Shubassi G, Bernstein KA, et al. (2011) HDACs link the DNA damage response, processing of double-strand breaks and autophagy. Nature 471: 74-79.

77. Potenski CJ, Klein HL (2011) Molecular biology: The expanding arena of DNA repair. Nature 471: 48-49. 